\title{
HIGH-RESOLUTION OBSERVATIONS OF VARIABLE RADIO SOURCES
}

\author{
A. T. MOFFET \\ California Institute of Technology, Pasadena, Calif., U.S.A. \\ and \\ J. GUBBAY \\ University of Adelaide, Adelaide, South Australia \\ and \\ D. S. ROBERTSON and A. J. LEGG \\ Weapons Research Establishment, Salisbury, South Australia
}

\begin{abstract}
Preliminary results are reported of a long-term program to test models of variable radio sources by direct observations of diameter changes. Although further confirmation is needed, increase in angular diameter attributed to a centimetric component of 3C 279 found in 1966 implies relativistic expansion with $\alpha \gtrsim 2$.

In order to sort out events taking place in variable sources it is necessary to observe frequently and at many wavelengths, especially at short wavelengths.
\end{abstract}

This is a progress report on a long-term program designed to test possible models of variable radio sources by direct observation of diameter changes of components of these sources. The observations are made with an independent-local-oscillator tape recorded interferometer using stations of the JPL-NASA Deep Space Network in California and Australia. The wavelength is $13 \mathrm{~cm}$, and the spacing between stations is about $8 \times 10^{7}$ wavelengths, giving a resolution of the order of $0.001 \mathrm{sec}$ of arc. The hardware used is all part of the standard station complement of equipment, so the only logistical problem is that of transporting the California tapes to Adelaide, where all the analysis is done on a CDC 6400 computer.

We have observed five sources in 1967 November, 1968 May and 1969 June. Concurrently with the last of these observations about 80 sources were observed with more sensitive equipment by the National Radio Astronomy Observatory - Caltech group (Kellermann et al., 1970). An improvement in the bandwidth of our recordings increased our sensitivity and permitted us to observe 25 sources in 1969 December and 1970 June. We expect to re-observe this group of sources approximately each six months. The sources were chosen from those which showed the strongest fringes in the 1969 June NRAO-Caltech observations.

Substantial changes in both total flux density and in the correlated flux density over the California-Australia baseline have been noted for many of the sources. When more observations have been made it should be possible to test the application of the expanding source model to many events. The best case for a test so far is in 3C 279. In this source the flux density has increased by 3 flux units between 1967 
November and 1969 December (our own observations and Nicolson, 1971). Over this same time the correlated intensity has increased by only $2.5 \mathrm{f}$.u. with a lower rate of increase than the flux density, particularly in the 1969 June to December interval. The increase in the flux density seems to be related to an expanding component first seen at short centimeter wavelengths in 1966 (Kellermann and Pauliny-Toth, 1968). It appears as if this component is now becoming resolved, implying that its angular diameter has reached about $0.001 \mathrm{sec}$ of arc. If subsequent observations confirm this, it will imply a relativistic expansion with $\gamma \sim 2$ (Rees, 1967).

In an earlier report on this work (Gubbay et al., 1969) we claimed evidence for a relativistic expansion in $3 \mathrm{C}$ 273. This claim was based on an identification of a flux density decrease in 3C 273 with an expanding component seen in 1966 at short wavelengths. A more detailed comparison of the data on flux density vs time at several wavelengths shows that this identification is probably not correct, since the expanding component would have a peak flux density at $13 \mathrm{~cm}$ of less than one flux unit (Epstein, 1971; more or less this same result was also communicated to ATM by Dr M. Walmsley). This serves to point up the need for continued monitoring of flux densities of variable sources at many wavelengths if we are to be able to sort out the events taking place in these objects. It is clear that repeated interferometer observations should also be made at other wavelengths and at several spacings. In particular, the expanding components will be much more intense at wavelengths of 2 or 3 centimeters, and observations in that range would be very desirable.

\section{Acknowledgements}

We are indebted to the staff of the various stations of the Deep Space Network for their assistance in these observations and to NASA and JPL for permission to use their facilities.

\section{References}

Epstein, E. E.: 1971, this volume, p. 227.

Gubbay, J., Legg, A. J., Robertson, D. S., Moffet, A. T., Ekers, R. D., and Seidel, B.: 1969, Nature 224, 1094.

Kellermann, K. I. and Pauliny-Toth, I. I. K.: 1968, Ann. Rev. Astron. Astrophys. 6, 417.

Kellermann, K. I., Clark, B. G., Jauncey, D., Cohen, M. H., Shaffer, D., Moffet, A. T., and Gulkis, S. : 1970, Astrophys. J. 161, 803.

Nicolson, G.: 1971, this volume, p. 224.

Rees, M.: 1967, Monthly Notices Roy. Astron. Soc. 135, 345. 